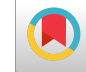

\title{
Nocardia cyriacigeorgica: A Case of Disseminated Infection in an Immunocompromised Patient
}

\author{
Soudabeh Aghajanpour, ${ }^{1}$ Davood Yadegarynia, ${ }^{1,}$ Zahra Arab-Mazar, ${ }^{1}$ and Hossein Dabiri ${ }^{1}$ \\ ${ }^{1}$ Infectious Diseases and Tropical Medicine Research Center, Shahid Beheshti University of Medical Sciences, Tehran, IR Iran \\ ${ }^{2}$ Department of Microbiology, School of Medicine, Shahid Beheshti University of Medical Sciences, Tehran, IR Iran \\ "Corresponding author: Davood Yadegarynia, Infectious Diseases and Tropical Medicine Research Center, Shahid Beheshti University of Medical Sciences, Tehran, IR Iran. Tel: \\ +98-2122439963, Fax: +98-2122439964, E-mail: d.yadegarynia@gmail.com
}

Received 2017 February 26; Revised 2017 March 07; Accepted 2017 March 14.

\begin{abstract}
Introduction: Nocardia cyriacigeorgica is a common environmental organism isolated from different clinical samples in Europe, Asia, and North America, predominantly from respiratory samples, but also from samples from several other sites.

Case Presentation: In the current paper a disseminated Nocardia cyriacigeorgica infection in an immunocompromised patient was reported. The patient was admitted to the under study hospital with multiple cutaneous abscess such as lesion and paraspinal abscess developed to brain abscess.

Conclusions: Nocardia cyriacigeorgica was isolated from a sample of skin lesion based on phenotypic properties and sequencing method. Limited cases of Nocardia cyriacigeorgica in Iran are reported.
\end{abstract}

Keywords: Immunocompromised Patient, Brain Abscess, Linezolid, Nocardia Infection

\section{Introduction}

Nocardiosis is a sever opportunistic infection in the last 2 decades due to the recent increase in the number of immunocompromised patients that occurred from advances in transplantation, cancer, chemotherapy, acquired immune deficiency syndrome (AIDS), and immunosuppression therapy in rheumatologic diseases (1). The causative organism of nocardiosis is Nocardia species; they are aerobic, catalase positive, Gram-variable to Grampositive and partially acid fast bacteria and are generally slow growing, non-motile bacilli with branching filaments. These soil-born ubiquitous actinomycetes often cause localized or disseminated infection. Despite the relative variety of Nocardia spp., as a cause of cerebral infection, the brain is the most frequent non-pulmonary site involved in disseminated nocardiasis (2). Disseminated nocardiosis is defined as a Nocardia infection in 2 or more non-contiguous sites.

Nocardia cyriacigeorgica is a recently identified species. It is isolated from clinical samples in Europe, Asia, and North America, predominantly from respiratory samples, but also from several other sites (3). The current report describes a disseminated nocardiosis caused by Nocardia cyriacigeorgica in an immunosuppressed patient. The current case is the 3rd report of N. cyriacigeorgica infection in Iran
(4).

\section{Case Report}

A 61-year-old male with the history of diabetes mellitus and rheumatoid arteritis was admitted in May 2015 to Khatam Al-Anbia Hospital, Tehran, Iran, with complaints of multiple cutaneous abscess lesions and right side weakness with lumbar pain.

He had been diagnosed with rheumatoid arteritis and vacuities 5 months earlier and was receiving immunosuppression therapy consisted of azathioprine (50 mg daily), oral prednisolone (75 mg daily), and methotrexate (7.5 mg weekly). On physical examination, he was lethargic and decreased force of right sided extremity muscles than the left side was observed. Also, lumbosacral spinal tenderness and multiple cutaneous abscess lesions $0.5-1.5 \mathrm{~cm}$ in diameter in thorax, abdomen, and upper and lower limbs were observed (Figure 1). Under examination, the vital signs were unremarkable at the time of admission:

His body temperature was $38.3^{\circ} \mathrm{C}$, pulse 93 beat/minute, blood pressure $115 / 75 \mathrm{mmHg}$, and respiratory rate 18 breath/minute. Laboratory testing revealed hemoglobin (Hb) $13.4 \mathrm{~g} / \mathrm{dL}$, total white blood cell count $(\mathrm{WBC}) 10.93 \times 10^{3} / \mathrm{L}$, and platelet count $255 \times 10^{3} / \mathrm{L}$. The

Copyright ( $)$ 2018, International Journal of Infection. This is an open-access article distributed under the terms of the Creative Commons Attribution-NonCommercial 4.0 International License (http://creativecommons.org/licenses/by-nc/4.0/) which permits copy and redistribute the material just in noncommercial usages, provided the original work is properly cited. 


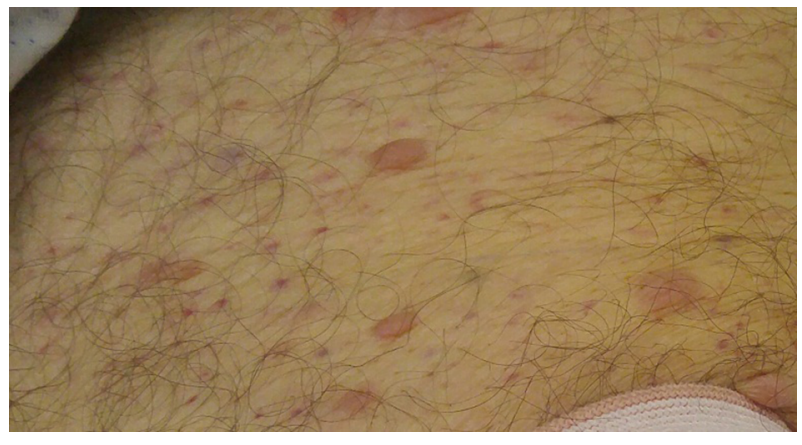

Figure 1. Nodule Presentation

erythrocyte sedimentation rate (ESR) was noted to be elevated at $76 \mathrm{~mm} / 1 \mathrm{st}$ hour.

Due to the lethargic condition with right sided weakness, brain computed tomography (CT) scan with intravenous (IV) contrast was done that revealed a mass lesion with cerebral abscess. The lumbosacral magnetic resonance imaging (MRI) was performed because of spinal tenderness, and paraspinal abscess in lumbosacral area was observed (Figures 2 and 3 ).

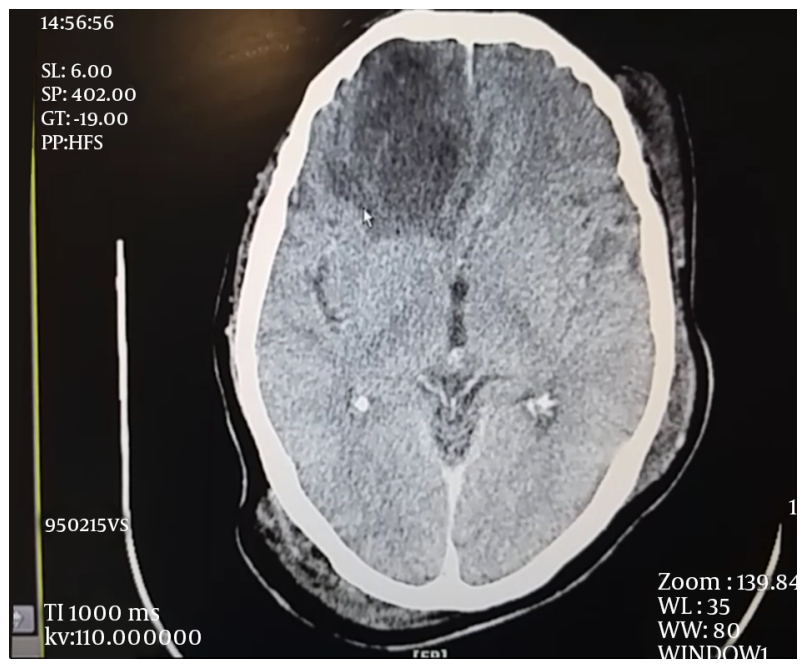

Figure 2. CT Scan at Presentation

The patient was treated with trimethoprim/sulfamethoxazole (TMP/SMX) (160/800 mg), and ceftriaxone and imipenem ( $1 \mathrm{~g}$ IV) due to cerebral abscess and multiple cutaneous abscess lesions and the history of immunosuppression therapy.

Due to suspicion of Nocardia infection, cutaneous abscess culture was sent to laboratory and vital signs and red flags were precisely controlled; 5 days later, culture anal-

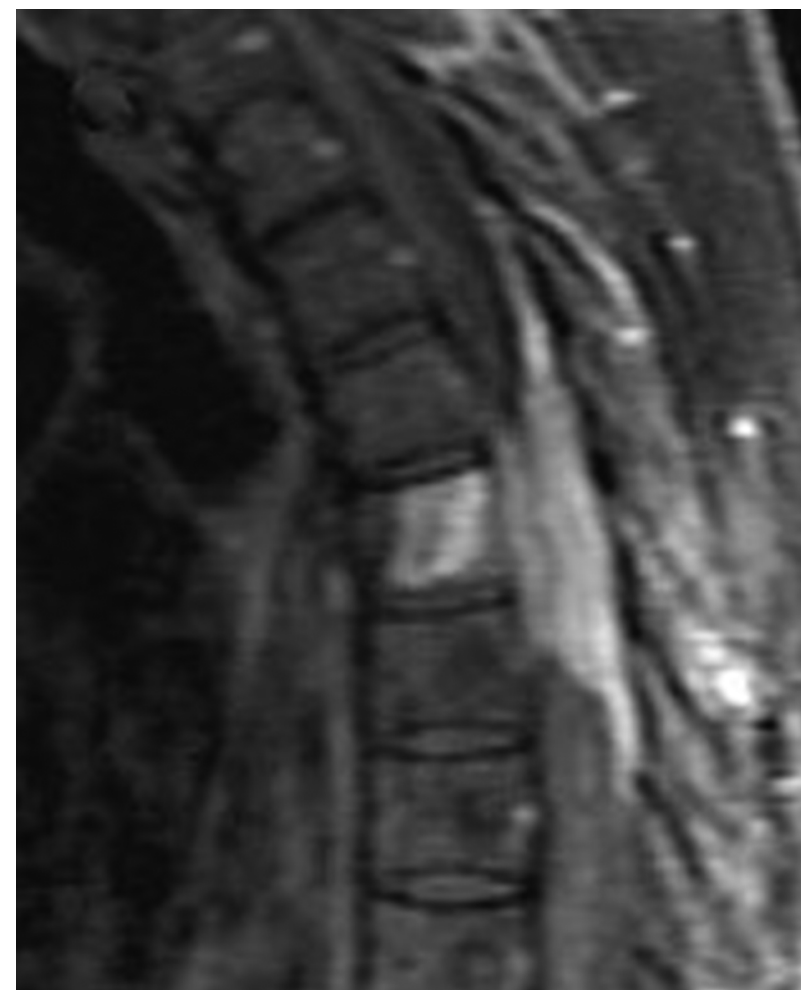

Figure 3. Paraspinal Abcess

ysis of cutaneous yielded Gram-positive filament bacilli identified as Nocardia sp.

Despite combination therapy of this disseminated Nocardia sp. with imipenem, ceftriaxone, and TMP/SMX, the patient deteriorated while on antibiotic therapy and his level of consciousness dropped. There was no change in the new brain CT scan (Figure 4). Chest X-ray showed infiltration in the lung base and bilateral pleural effusion (Figure 5). For this reason, linezolid (600 mg IV twice a day) was added to the previous treatment regimen.

Unfortunately, the patient rapidly died. Post mortem examinations of isolated Nocardia sp. based phenotypic properties and sequencing method indicated Nocardia cyriacigeorgica as the cause of disease. The initial isolation of the current study sample was identified by the phenotypic properties such as modified acid fast growth in lysozyme, and hydrolyzing tyrosine, xanthine, hypoxanthine and uric acid. To identify more precisely, polymerase chain reaction (PCR) was performed on 16s-rRNA of the isolated bacteria. The 16s-rRNA sequences were recorded in GenBank of NCBI. The16s-rRNA sequences of Nocardia cyriacigeorgica in the current case were submitted to GenBank, and closely compared with those of the NCBI regis- 


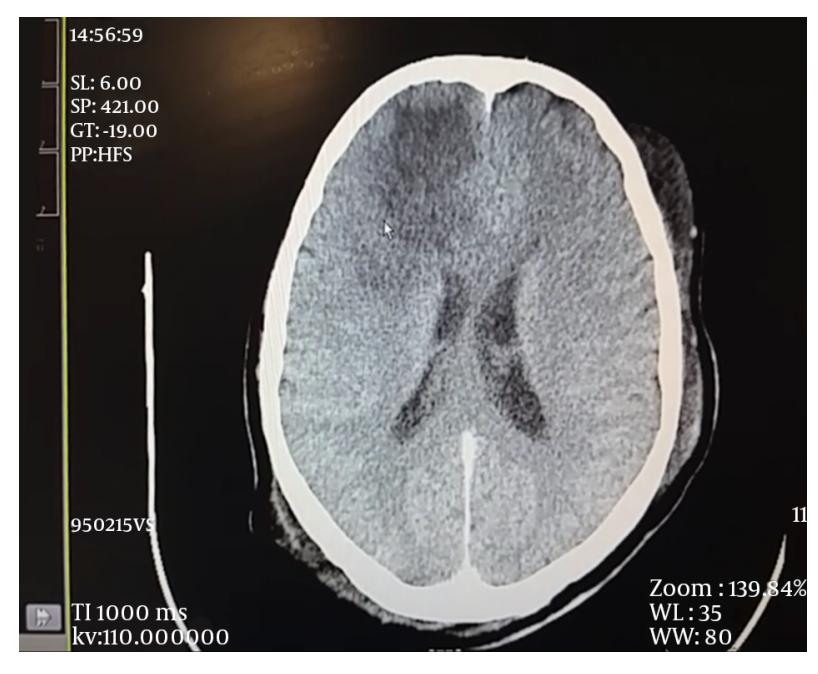

Figure 4. CT Scan After Treatment Demonstrated Improvements.

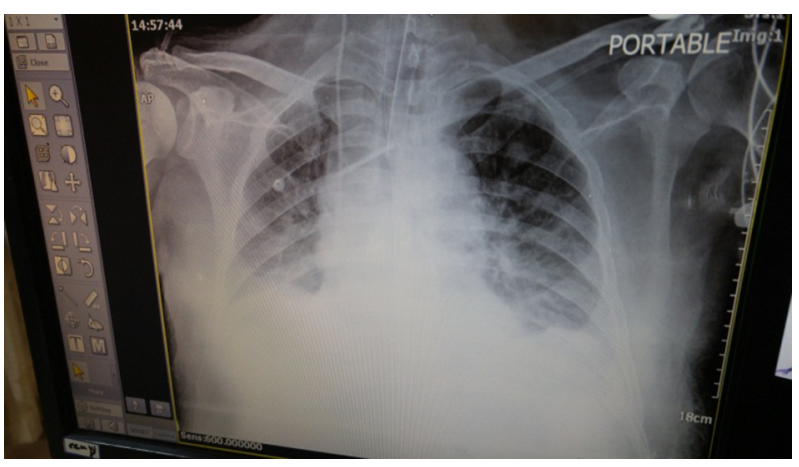

Figure 5. Chest X-Ray Showed Infiltration in the Lung Base and Bilateral Pleural Effusion.

tered type ( $N$. cyriageorgica DSM44434T); and 100\% similarity with this strain and $0 \%$ gap in this isolate was found.

\section{Discussion}

Nocardia cyriageorgica was first identified in 2001 by Yassin et al. (5) as a novel species differing from the previously described members of genus Nocardia in terms of molecular and biomedical characteristics. A study by Convill et al. based on DNA-DNA homology, indicated that $N$. cyriageorgica was indeed a member of $N$. astroides drug patter VI. In the Southern USA N. asteroids drug type VI is the most frequent reported Nocardia sp. This species of Nocadia is found primarily in soil, but can also inhibit humans and is sometimes found in water habitats (6). Neocardiacyriageorgica, similar to other species of Nocardia, is considered an opportunistic pathogen that affects immunosuppressed patients who are particularly susceptible to Nocardia cyriacigeorgica (7). Nocardia cyriacigeorgica could be an etiologic agent of Parkinson disease due to its ability to spread to the brain via the blood stream (8). Dry and windy areas are the primary location for the infection, since the winds enhance the relocation of the microbe (8). Nocardia cyriacigeorgica and type VI strains of Nocardia spp. have a same antibiogram susceptibility pattern and are typically susceptible to sulfonamide, amikacin, linezolid, imipenem, and broad spectrum cephalosporins (9), while remaining resistant to clarithromycin and penicillin, with the exception of a few strains of $N$. cyriacigeorgica that are reported susceptible to ciprofloxacin (10).

The current case results may help to better identify $N$. cyriacigeorgica and its ability to cause disseminated Nocardia infection. The lungs are the primary site of Nocardia cyriacigeorgica infection (11). Neocardia cyriageorgica was originally thought to belong to NAC, due to differences in antibiotic susceptibility patterns; now it is considered a separate entity with the advent of newer molecular diagnostic techniques such as 16s-rRNA gene (12) or matrix-assisted laser depression. It is reported that ionization time to combat invasive infection caused by N.cyriageorgica is increasing.

\section{References}

1. Uttamchandani RB, Daikos GL, Reyes RR, Fischl MA, Dickinson GM, Yamaguchi E, et al. Nocardiosis in 30 patients with advanced human immunodeficiency virus infection: clinical features and outcome. Clin Infect Dis. 1994;18(3):348-53. [PubMed: 8011814].

2. Hooper DC, Pruitt AA, Rubin RH. Central nervous system infection in the chronically immunosuppressed. Medicine (Baltimore). 1982;61(3):166-88. [PubMed: 7043156].

3. Cargill JS, Boyd GJ, Weightman NC. Nocardia cyriacigeorgica: a case of endocarditis with disseminated soft-tissue infection. J Med Microbiol. 2010;59(Pt 2):224-30. doi: 10.1099/jmm.0.011593-0. [PubMed: 19797466].

4. Shojaei H, Hashemi A, Heidarieh P, Eshraghi S, Khosravi AR, Daei Naser A. Clinical isolation of Nocardia cyriacigeorgica from patients with various clinical manifestations, the first report from Iran. Med Mycol J. 2011;52(1):39-43. [PubMed: 21441712].

5. Yassin AF, Rainey FA, Steiner U. Nocardia cyriacigeorgici sp. nov. Int J Syst Evol Microbiol. 2001;51(Pt 4):1419-23. doi: 10.1099/00207713-51-41419. [PubMed: 11491341].

6. Beaman BL, Beaman L. Nocardia species: host-parasite relationships. Clin Microbiol Rev.1994;7(2):213-64. [PubMed: 8055469].

7. Brown-Elliott BA, Brown JM, Conville PS, Wallace RJ. Clinical and laboratory features of the Nocardia spp. based on current molecular taxonomy. Clin Microbiol Rev. 2006;19(2):259-82. doi: 10.1128/CMR.19.2.259-282.2006. [PubMed: 16614249].

8. Hubble JP, Cao T, Kjelstrom JA, Koller WC, Beaman BL. Nocardia species as an etiologic agent in Parkinson's disease: serological testing in a case-control study. J Clin Microbiol. 1995;33(10):2768-9. [PubMed: 8567923].

9. Conville PS, Witebsky FG. Organisms designated as Nocardia asteroides drug pattern type VI are members of the species Nocardia cyr- 
iacigeorgica.JClin Microbiol.2007;45(7):2257-9. doi: 10.1128/JCM.0013307. [PubMed: 17475753].

10. Barnaud G, Deschamps C, Manceron V, Mortier E, Laurent F, Bert F, et al. Brain abscess caused by Nocardia cyriacigeorgica in a patient with human immunodeficiency virus infection. J Clin Microbiol. 2005;43(9):4895-7. doi: 10.1128/JCM.43.9.4895-4897.2005. [PubMed: 16145170].

11. Hirayama T, Takazono T, Horai Y, Tashiro M, Saijo T, Kosai K, et al. Pulmonary Nocardiosis Caused by Nocardia concava with a
Literature Review. Intern Med. 2016;55(9):1213-7. doi: 10.2169/internalmedicine.55.6228. [PubMed: 27150883].

12. Fatahi-Bafghi M, Heidarieh P, Rasouli-Nasab M, Habibnia S, HashemiShahraki A, Eshraghi SS. Comparison of restriction enzyme pattern analysis and full gene sequencing of 16S rRNA gene for Nocardia species identification, the first report of Nocardia transvalensis isolated of sputum from Iran, and review of the literature. Antonie Van Leeuwenhoek. 2016;109(10):1285-98. doi: 10.1007/s10482-016-0746x. [PubMed: 27613736]. 